\title{
Detrusor Myoplasty for Acontractile/Hypocontractile Bladder in Spinal Cord Injury Patients
}

Pawan Agarwal ${ }^{1 *}$, Dhananjaya Sharma ${ }^{2}$

Plastic Surgery Unit, Department of Surgery, NSCB Government Medical College, Jabalpur, MP, India

\section{Article Info}

\section{Article Notes}

Received: April 12, 2018

Accepted: August 14, 2018

\section{${ }^{*}$ Correspondence}

Dr Pawan Agarwal, 292/293, Napier Town, Jabalpur, 482001, MP, India; E-mail: drpawanagarwal@yahoo.com.

(c) 2018 Agarwal P. This article is distributed under the terms of the Creative Commons Attribution 4.0 International License

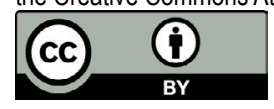

\section{Keywords}

Detrusor-Myoplasty

Bladder; Neurogenic

Spinal Cord Injury

Urodynamics

Rectus Abdominis Muscle
Abstract

Acontractile bladder is very common after spinal cord injury which may lead to morbidity and even renal failure. The standard treatment options to facilitate bladder emptying include clean intermittent catheterization $(\mathrm{CIC})$ or indwelling catheter (IC), which is also not free of complications. The purpose of this article is to review the treatment options for acontractile bladder; especially feasibility and effectiveness of muscle wrap around urinary bladder to improve voluntary voiding. We performed Rectus Abdominis Detrusor Myoplasty (RADM) in 5 patients of acontractile bladder following spinal cord injury. Postoperatively, all 5 patients could void urine immediately after removal of per-urethral catheter after 3 weeks. After RADM, Post Void Residual Volume, Bladder Contractility Index, Detrusor Pressure and urine flow rate $(V$-max) improved significantly $(p<0.05)$ in all patients. Rectus Abdominis Detrusor Myoplasty is a promising surgical option in a patient having hypocontractile or acontractile urinary bladder. In this mini review RADM and other treatment options for acontractile bladder have been reviewed.

\section{Introduction}

Urinary bladder dysfunction is very common after spinal cord injury and leads to multiple morbidities in these patients. Patients having spinal injury below the level of $12^{\text {th }}$ thoracic vertebra (D12) have a hypo-contractile or acontractile bladder while those who have an injury above the level of D12 may have spastic or mixed type dysfunction of bladder. Multiple causes at any level between the brain and the bladder can lead to acontractile bladder and these include congenital malformations, acquired infections, inflammatory or autoimmune diseases, central or peripheral nerve injuries secondary to trauma or degenerative diseases, detrusor myopathy, and idiopathic causes ${ }^{1-3}$. Acontractile bladder leads to recurrent urinary tract infection (UTI), vesico-ureteric reflux, stones formation, back pressure changes and deteriorating renal function ${ }^{4}$. The standard treatment options to facilitate bladder emptying include clean intermittent catheterization (CIC) or indwelling catheter (IC) in the form of suprapubic/urethral catheters, and urinary diversions such as ileal or colonic conduits ${ }^{5}$. Both, CIC and IC, have their well-known attendant morbidities like recurrent UTI, epididymo-orchitis, urethral trauma and stricture, bladder injury, urolithiasis, deteriorating renal function and adverse effects on patient's quality of life ${ }^{6,7}$. The purpose of this article is to review the treatment options for acontractile bladder; especially feasibility and effectiveness of muscle wrap around urinary bladder to improve voluntary voiding.

Methodology for review - Literature search was made via PUBMED and Google Scholar, using detrusor-myoplasty, bladder, neurogenic, spinal cord injury, urodynamics, rectus abdominis muscle key words and relevant articles were accessed. The cross references of full articles were also accessed and reviewed. 


\section{Historical Background}

Historically, techniques to improve bladder contractility/ voiding effectiveness include electric stimulation of bladder or its nerve supply, manipulation of bladder neck (pharmacological or surgical weakening) or strengthening of detrusor by a muscle wrap or sling. But their inconsistent efficacy in improving detrusor contractility and side effects limits their use in clinical practice $^{8}$.

\section{Review of Literature}

In early 1990s, von Heyden and Chancellor described for the first time the successful use of the latissimus dorsi (LD) and Rectal Abdominis (RA) muscle respectively in animal experiments in dogs. However, these dogs were able to evacuate only $50 \%$ of urine ${ }^{9-11}$. These elegant studies showed the principle, feasibility and technique of detrusor-myoplasty (DMP) and free LD myoplasty which can augment lost detrusor function with minimal morbidity and acceptable results. Stenzl in their experimental studies in dogs confirmed improved bladder function after LD detrusor-myoplasty (LDDM) and suggested refinement in the technique ${ }^{12,13}$. The electrically stimulated detrusor myoplasty in dogs worked well acutely to increase vesical pressure sufficient to empty the bladder, but the chronically stimulated myoplasty did not maintain efficient bladder emptying primarily due to electrode problems ${ }^{\mathbf{1 4}}$.

In 1985, Messing published the first report of functional bladder augmentation using bilateral pedicled rectus femoris muscle flaps ${ }^{15}$. This was followed by reports of DMP technique using rectus abdominis muscle as a pedicled flap ${ }^{16,17}$. However, both techniques did not seem capable of building up or sustaining adequate pressure needed for micturition. Stenzl et al. published in 1998 their first experience with LDDM in three patients with acontractile bladder ${ }^{18}$; followed by a series of publications showing long-term success ${ }^{1,19-21}$.

A recent option for acontractile bladder in spina bifida is lumbar-to-sacral nerve rerouting. This technically demanding surgery involves joining the L5 nerve to the S2/ S3 ventral roots; it has met with limited success ${ }^{22}$.

\section{Case Series}

We have published our technique and results with RADM in 5 patients ${ }^{23}$. We evaluated 20 consecutive patients with spinal cord injury to determine the type of bladder dysfunction only 5 patients were found suitable for rectus abdominis detrusor myoplasty (RADM) after preoperative urodynamic study.

All 5 patients (median age 38.31years, range 30-50 years, all male) had acontractile bladder due to spinal cord injury below D12 level sustained 1-3 years back. 4/ 5 patients had indwelling catheter and 1 patient was on CIC. They had 6-8 episodes of UTI every year. UTI was documented with positive urine culture.

Mean operating time was 158 minutes (range 140-180 minutes). Length of hospital stay was 9.6 days (range 7-12). Mean follow-up was 15.3 months (range 6-24 months).

Postoperatively, all 5 patients could void urine (uninterrupted flow of urine with $13 \mathrm{ml} / \mathrm{s}$ flow rate) immediately after removal of per-urethral catheter after 3 weeks. After RADM, Post Void Residual Volume, Bladder Contractility Index, Detrusor Pressure and urine flow rate (V-max) improved significantly $(\mathrm{p}<0.05)$ in all patients. All the data was collected and entered in Microsoft excel sheet and then transferred to IBM-SPSS software version 21.0 for analysis. Data was analysed using ANOVA repeated measures statistical tests. P-value $<0.05$ was taken as level of significance.

There were no early or late complications like flap failure, muscular deficit at the donor site, chronic pain or hernia. Back pressure changes were not seen on ultrasound at 6 month after RADM. At 1-year good blood flow in muscle was seen on Doppler and good contraction was seen on USG and EMG in 4 patients (one patient has completed follow-up of only 6 months). These patients no longer have recurrent UTIs as documented by negative urine culture.

\section{Discussion}

It is estimated that more than 250,000 people are living with spinal injuries in the USA. The National Spinal Cord Injury Statistical Centre reports 40 new cases per million of the population per year ${ }^{24}$. In India; incidence of spinal injuries is estimated to be around 20 per million with 2,500 fresh cases added every year, often as a result of road accidents $^{25}$.

The ideal muscle for bladder myoplasty should have appropriate tissue architecture, anatomical arrangement, fiber length for excursion, sufficient cross section and muscle mass for exerting force ${ }^{26}$. Currently, only two muscles Rectus abdominis (pedicle flap) and LD (free flap) are used successfully for detrusor myoplasty. However, free LD flap is a major undertaking; long surgery requires two specialist teams and has a steep learning curve. It requires micro-surgical skills and can be done only in tertiary referral centers. Additionally, the transplanted LD muscle flap takes time for re-innervation of muscle and in best of the hands there is almost 5-10 \% failure rate for free flap. It also creates a large thoracic wall defect, and this assumes greater importance in paraplegic patients who rely on their upper extremities and torso strength for mobility ${ }^{27}$.

The use of the rectus abdominis is a simple surgical procedure and has no risk of failed re-innervation and loss of vascularity of flap. An innervated muscle is transposed, hence there is no 'waiting period' for the muscle to start 
working and early results are obtained in these patients. Most of the patients are able to initiate bladder emptying without extensive training due to synergistic nerve supply and increase in bladder intra-luminal pressure which triggers the sphincter relaxation ${ }^{18}$.

RADM has been 'theoretically' criticized on the basis that segmental nerve supply varies and proximal part of the muscle can get denervated in the process of mobilization which in the long term may lead to reduction in power, contracture of muscle and ultimately poor results. We overcame this potential problem of partial muscle denervation by anastomosing two intercostal nerves from upper part of muscle to opposite intercostal nerves ${ }^{23}$. Additionally, RA is an expandable muscle and its use for detrusor myoplasty does not cause any functional deficit. Long-term follow-up of patients after RA muscle transfer for breast reconstruction has demonstrated no significant deficit at donor site ${ }^{28}$. An additional benefit of muscle wrap is that it can modify the bladder shape and capacity; a tight wrap will decrease the volume of the over distended atonic bladder. While a loose wrap may serve to maintain a larger bladder capacity ${ }^{18}$. But at the same time the resting muscle length during transfer should be maintained because efficiency of skeletal muscle depends on its resting tension ${ }^{29}$.

The indications for the detrusor myoplasty include bladder acontractility, unsuccessful sacral neuromodulation and complications/non-feasibility of clean intermittent catheterization. Contraindications for the detrusor myoplasty include patients with upper motor neuron lesion, intact spinal cord micturition center and spinal roots (candidate for sacral neuromodulation), bladder outlet obstruction, incontinence, improving bladder function, defective innervation of the rectus abdominis muscle, previous surgery in the pelvic region, and bladder capacity $<400 \mathrm{ml}$.

However, a word of caution is mandatory: A study that reviewed the outcomes of 101 patients who had been on CIC for an average of 5 years found that the majority of patients were able to perform self-catheterization easily, without an impact on their daily activities, so it would be inappropriate to assume that all patients on CIC are candidates for Detrusor Myoplasty ${ }^{30,31}$.

\section{Conclusion}

Detrusor myoplasty appears to be a promising surgical option in selected patients with acontractile/ hypocontractile bladder to restore the bladder function and improve quality of life. The value and effect of detrusor myoplasty on patient quality of life should be weighed against simpler measures such as CIC or neuromodulation (when possible) techniques and the exact indications for this procedure need to be defined.

\section{The Future}

Apart from the restoration of deficient detrusor function, transfer of a muscle flap may be combined with tissue engineering to serve as a basis for non-intestinal bladder augmentation and substitution. Substitution of the defective smooth-muscle lining an internal organ by means of neurovascular free striated-muscle transfer may prove to be useful in a number of conditions ${ }^{32}$.

\section{References}

1. Stenzl A, Strasser H, Klima G, et al. Reconstruction of the lower urinary tract using autologus muscle transfer and cell seeding: current status and future perspectives. World J Urol. 2000; 18: 44-50.

2. Ninkovic M, Stenzl A, Gakis G, et al. The latissimus dorsi detrusor myoplasty for functional treatment of bladder acontractility. Clin Plast Surg. 2012; 39: 507-12.

3. Van Koeveringe G, Rademakers K, Stenzl A. Latissimus dorsi detrusor myoplasty to restore voiding in patients with an acontractile bladder - fact or fiction? Curr Urol Rep. 2013; 14: 426-34.

4. Shingleton WB, Bodner DR. The development of urologic complications in relationship to bladder pressure in spinal cord injured patients. J Am Paraplegia Soc. 1993; 16: 14-7.

5. Ginsberg DA. Bladder acontractility: detrusor myoplasty and other options. Nat Rev Urol. 2011; 8: 185-6.

6. Madjar S, Appell RA. Impaired detrusor contractility: anything new? Curr Urol Rep. 2002; 3: 373-7.

7. Liu CW, Attar KH, Gall A, et al. The relationship between bladder management and health-related quality of life in patients with spinal cord injury in the UK. Spinal Cord. 2010; 48: 319-24.

8. Madjar S, AppellRA.Impaired detrusor contractility: anything new. CurrUrol Rep. 2002; 3: 373-7.

9. von Heyden B, Anthony JP, Kaula N, et al. The latissimus dorsi muscle for detrusor assistance: functional recovery after nerve division and repair. J Urol. 1994; 151: 1081- 87.

10. Chancellor MB, Rivas DA, Acosta R, et al. Detrusor-myoplasty, innervated rectus muscle transposition study, and functional effect on the spinal cord injury rat model. NeurourolUrodyn. 1994; 13: 547-57.

11. von Heyden B, Anthony JP, Brock GB, et al. The latissimus dorsi bladder myoplasty to assist detrusor function. Urol Res. 1998; 26: 215-21.

12. Stenzl A, Ninkovic M, Willeit J, et al. Free neurovascular transfer of latissimus dorsi muscle to the bladder I Experimental studies. J Urol1. 1997; 157: 1103-8.

13. Stenzl A, Ninkovic M. Restoring voluntary urinary voiding using a latissimus dorsi muscle free flap for bladder reconstruction. Microsurgery. 2001; 21: 235-40.

14. Van Savage JG, Perez-Abadia G, Palanca LG, et al. Comparison of the experience with acute and chronic electrically stimulated detrusor myoplasty. NeurourolUrodyn. 2002; 21: 516-21.

15. Messing EM, Dibbell DG, Belzer FO. Bilateral rectus femoris pedicle flaps for detrusor augmentation in the prune belly syndrome. J Urol. 1985; 134: 1202-5.

16. Zhang YH, Shao QA, Wang JM. Enveloping the bladder with displacement of flap of the rectus abdominis muscle for the treatment of neurogenic bladder. J Urol. 1990; 144: 1194-5

17. Chancellor MB, Rivas DA, Salzman SK. Detrusor-myoplasty to restore micturition. Lancet. 1994; 343: 669.

18. Stenzl A, Ninkovic M, Kolle D, et al. Restoration of voluntary emptying 
of the bladder by transplantation of innervated free skeletal muscle. Lancet. 1998; 351: 1483- 5.

19. Stenzl A. Free neurovascular transfer of latissimus dorsi muscle for the treatment of bladder acontractility: II. Clinical results. IntBraz J UrolOff J BrazSoc Urol. 2003; 29: 179-80.

20. Ninkovic M, Stenzl A, Schwabegger A, et al. Free neurovascular transfer of latissimus dorsi muscle for the treatment of bladder acontractility: II. Clinical results. J Urol. 2003; 169: 1379-83.

21. Gakis G, Ninkovic M, van Koeveringe GA, et al. Functional detrusor myoplasty for bladder acontractility: long-term results. JUrol. 2011 185: 593-9.

22. Peters KM, Girdler B, Turzewski C, et al. Outcomes of lumbar to sacral nerve rerouting for spina bifida. J Urol. 2010; 184: 702-707.

23. Agarwal P, Husain S, Wankhede $S$, et al. Rectus abdominis detrusor myoplasty for acontractile/hypocontractile bladder in spinal cord injury patients: Preliminary report. J Plast Reconstr Aesthet Surg. 2017 Dec 14; pii: S1748-6815(17)30514-4. doi: 10.1016/j. bjps.2017.12.015. [Epub ahead of print]]

24. Taweel WA, Seyam R. Neurogenic bladder in spinal cord injury patients. Res Rep Urol. 2015; 10: 785-99.

25. Dhar A. Few steps forward. The Hindu. 2013. Available from: http://www.thehindu.com/sci-tech/health/few-stepsforward/ article4978239.ece [Accessed 5 May 2017]

26. ManktelowR,Zuker RM.Theprinciples offunctioningmuscletransplantation: applications to the upper arm. Ann Plast Surg. 1989; 22: 275.

27. He J, Xu H, Wang T, et al. Treatment of complex ischial pressure sores with free partial lateral latissius dorsi musculo-cutaneous flaps in paraplegic patients. J Plast Reconstr Aesthet Surg. 2012; 65: 634-39.

28. Lejour M, Dome M. Abdominal wall function after rectus abdominis transfer. Plast Reconstr Surg. 1991; 87: 1054-68.

29. Spotnitz HM, Merker C, Malm JR. Applied physiology of the canine rectus abdominis: force-length curves correlated with functional characteristics of a rectus powered "ventricle." Potential for cardiac assistance. Trans Arn Soc Artif Intern Organs.1974; 20B: 747-55.

30. Kessler TM, Ryu G, Burkhard FC. Clean intermittent self catheterization: a burden for the patient. Neurourol Urodyn. 2009; 28: 18-21.

31. Ginsberg DA. Bladder acontractility: detrusor myoplasty and other options. Nat Rev Urol. 2011; 8: 185-6.

32. Oberpenning F, Meng J, Yoo JJ, et al. De novo reconstitution of a functional mammalian urinary bladder by tissue engineering. Nat Biotechnol. 1999; 17: 149-155. 